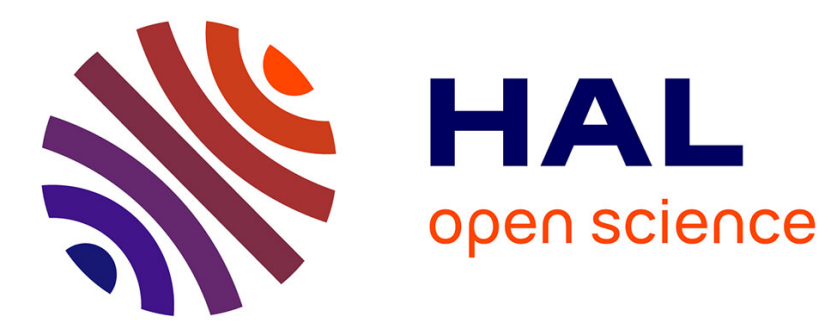

\title{
Performance Analysis of the Revisited Tone Mapped Quality Index for Tone Mapped HDR Images Evaluation
}

Ba Chien Thai, Anissa Mokraoui

\section{To cite this version:}

Ba Chien Thai, Anissa Mokraoui. Performance Analysis of the Revisited Tone Mapped Quality Index for Tone Mapped HDR Images Evaluation. International Conference on Telecommunications, Apr 2019, Hanoi, Vietnam. 10.1109/ICT.2019.8798770 . hal-02428263

\section{HAL Id: hal-02428263 https://hal.science/hal-02428263}

Submitted on 5 Jan 2020

HAL is a multi-disciplinary open access archive for the deposit and dissemination of scientific research documents, whether they are published or not. The documents may come from teaching and research institutions in France or abroad, or from public or private research centers.
L'archive ouverte pluridisciplinaire HAL, est destinée au dépôt et à la diffusion de documents scientifiques de niveau recherche, publiés ou non, émanant des établissements d'enseignement et de recherche français ou étrangers, des laboratoires publics ou privés. 


\section{Performance Analysis of the Revisited Tone Mapped Quality Index for Tone Mapped HDR Images Evaluation}

\author{
Ba Chien Thai \\ University of Science and Technology \\ 54 Nguyen Luong Bang Street, Lien Chieu District \\ Danang, Vietnam \\ tbchien@dut.udn.vn
}

\author{
Anissa Mokraoui \\ L2TI, Institut Galilée, Université Paris 13 \\ 99, Avenue Jean-Baptiste Clément \\ Villetaneuse, France \\ anissa.mokraoui@univ-paris13.fr
}

\begin{abstract}
This paper discusses the appropriate choice of the Tone Mapped Quality Index (TMQI) parameters. The TMQI metric has been developed to evaluate and compare the visual quality of the Tone Mapped (TM) High Dynamic Range (HDR) images. However, the parameters of this metric have been adjusted on a specific and reduced set of Tone Mapping Operators (TMO) and a restricted base of HDR images. The visual rendering evaluation of the TM images is then no longer consistent with the metric when new TMO (excluded from the initial training process) are used. To better adjust these parameters, discussions and experiments have been conducted on a large extension of the initial TMOs set and tone mapped HDR images dataset. The new parameters show a strong correlation between the revisited metric and the Mean Opinion Score (MOS).
\end{abstract}

Index Terms-High Dynamic Range image, Image Tone Mapping Operators, Human Visual System, Mean Opinion Score, Tone Mapped Quality Index.

\section{INTRODUCTION}

A High Dynamic Range (HDR) image contains both underexposed (i.e. very dark) and over-exposed (i.e. bright) areas in accordance with the real-world scene. Typical examples of HDR scenes are sunny outdoor scenes or views of an indoor room with objects visible outside the window. The dynamic ranges of several natural scenes have about 1 : 160 average contrast ratio. Outdoor scenes usually have a larger dynamic range, which can reach a contrast ratio of three orders of magnitude 1 : 1000 or sometimes even more. However, these HDR images cannot be visualized on standard Low Dynamic Range (LDR) display devices since their dynamic range is smaller than that of HDR images. The currently used display technologies are Cathode Ray Tube (CRT) or Liquid Crystal Displays (LCD), they are typically discretized at 8-bits and rarely at 10-bits per color channel. This means that colors shades are limited to 255. Moreover HDR display devices currently remain too expensive. Therefore many image Tone Mapping Operators (TMOs) have been developed. Their main concerns are to reduce the dynamic range (contrast, color gamut, details...) of those HDR images to the dynamic range of LDR display device while preserving, as much as possible, the appearance of the captured scene in terms of contrast and the overall impression of brightness and colors.

Many HDR image TMOs have been developed to convert HDR images into LDR images. However to measure the performance of these approaches, objective metrics are required not only to compare the visual rendering quality but also to provide a better understanding of the relationship between an operator and the image attributes. Indeed this can help in the development of TMOs more suited to a particular application such as an improved method for highlighting those parts of an image that are perceptually important to the Human Visual System (HVS).

The assessment of the Tone Mapped (TM) image is restricted either to no reference criteria, which were mostly trained and tested in different context, or to the specifically adjusted full reference metrics. All of the full reference image quality metrics assume that the dynamic range of the original and processed image are the same. However, in the case of TM HDR images, the dynamic range between the two versions is different (i.e. $\mathbf{I}_{H D R}$ is HDR image while $\mathbf{I}_{L D R}$ is LDR). That is why the development of objective metrics is not easy.

The main purpose of this paper is to choose one objective metric among many others that is best suited to our concerns, namely the visual quality evaluation of the TM HDR images with no HDR display device. To do so, this paper proceeds as follows. Section II introduces the well known objective metrics to assess the visual quality of TM HDR images. Section III analyzes the performance of these objective metrics when a large set of TMOs are applied to an extensive HDR image database. The metrics are compared to subjective assessments made by observers. The analysis of simulation results shows that none of the objective metrics is highly correlated to the Mean Opinion Score (MOS). However, the Tone Mapped Quality Index (TMQI) would seem to better meet our expectations although it is designed in a different context of experimentation of ours. It is then revised in section III-C with a large set of TMOs and an extensive HDR image database. Section IV confirms the performance of the revisited TMQI metric. Section V concludes this paper. 


\section{AN OVERVIEW OF OBJECTIVE VISUAL QUALITY METRICS FOR TONE MAPPED HDR IMAGES}

This section introduces some objective metrics often used to assess the visual quality of the tone mapped HDR images.

\section{A. Dynamic Range Independent Metric}

The first metric, called Dynamic Range Independent Metric (DRIM) [1], exploits the detection model from HDRVDP [2] which is calibrated on ModelFest dataset [24] to indicate the regions containing visible contrast in the HDR image and its TM version. The DRIM metric proposes three distortion maps showing the regions where the contrast is either lost (i.e. contrast change is perceivable in HDR but imperceivable in LDR image), amplified (the opposite case), or reversed (the polarity is changed - mostly caused by halo artifacts) by tone mapping process. This DRIM metric inherits high computational requirements and the necessity to specify viewing conditions and display parameters. Note that this metric is designed for visualization of the perceived distortions regarding the image contrast. As a result, it does not provide a single value for quality assessment. For this reason, it will not be considered in the performance analysis section.

\section{B. Tone Mapped image Quality Index}

Another HDR image tone mapping metric has been introduced by Yeganeh and Wang known as Tone Mapped image Quality Index (called TMQI1) [3]. It is composed of two parts: the Structural Fidelity $1(S F 1)$ part and Statistical Naturalness 1 ( $S N 1)$ part described below.

1) Structural Fidelity: $S F 1$ is a modified version of the Multi Scale Structural Similarity Index (MS-SSIM) [21]. For a patch $u$, it is given by:

$$
\begin{gathered}
S F 1\left(\mathbf{I}_{H D R}(u), \mathbf{I}_{L D R}(u)\right)= \\
\frac{2 \sigma^{\prime}\left(\mathbf{I}_{H D R}(u)\right) \cdot \sigma^{\prime}\left(\mathbf{I}_{L D R}(u)\right)+C_{1}}{\sigma^{\prime 2}\left(\mathbf{I}_{H D R}(u)\right)+\sigma^{\prime 2}\left(\mathbf{I}_{L D R}(u)\right)+C_{2}} \\
\times \frac{\sigma\left(\mathbf{I}_{H D R}(u), \mathbf{I}_{L D R}(u)\right)+C_{2}}{\sigma\left(\mathbf{I}_{H D R}(u)\right) \cdot \sigma\left(\mathbf{I}_{L D R}(u)\right)+C_{2}},
\end{gathered}
$$

where $\sigma^{\prime}($.$) is the standard deviation of the patch af-$ ter the nonlinear mapping; $\sigma\left(\mathbf{I}_{H D R}(u)\right), \sigma\left(\mathbf{I}_{L D R}(u)\right)$ and $\sigma\left(\mathbf{I}_{H D R}(u), \mathbf{I}_{L D R}(u)\right)$ are the local standard deviations and cross correlation between the two corresponding patches in HDR and LDR images, respectively; and $C_{1}, C_{1}$ are positive constants that make sure the equation not divided by zero.

Note that the luminance component is missing, compared the SSIM definition, but the structural element (i.e. the second fraction in equation (1)) remains the same.

The mapping is defined as follows:

$$
\sigma^{\prime}(\sigma)=\frac{1}{\sqrt{2 \pi} \theta_{\sigma}} \int_{-\infty}^{\sigma} \exp \left[-\frac{\left(x-\tau_{\sigma}\right)^{2}}{2 \theta_{\sigma}^{2}}\right]
$$

where

$$
\theta_{\sigma}(f)=\frac{\tau_{\sigma}(f)}{k},
$$

with $f$ being a spatial frequency and $k$ representing a constant obtained from Croziers law [25], typically ranging from 2.3 to 4 . The authors proposed to set $k=3$. The threshold for the signals standard deviation is given by:

$$
\tau_{\sigma}(f)=\frac{\bar{\mu}}{\sqrt{2} \times \lambda \times C S F(f)},
$$

where $\bar{\mu}$ is the mean intensity value (set to 128 by the authors) and $\lambda$ is a constant used to fit the physiological data. TMQI1 uses the CSF as introduced by Mannos and Sakrison [26] and fits to the data measured by Kelly [27]. The map of $S F 1$ is averaged on each scale and the final $S F 1$ index is obtained in the same way as in the case of MS-SSIM.

2) Statistical Naturalness: The second part of the metric, i.e. $S N 1$, does not need a reference. It is based on the assumption that naturalness of an image can be modeled by the probability distribution of the brightness and contrast (means and standard deviations) in natural gray-scale images. The means and standard deviations are modelled by a Gaussian $(\mathcal{N})$ and a Beta $(\mathcal{B})$ distribution respectively. The distributions are then given by $\mathcal{N}(115.94,27.99)$ and $\mathcal{B}(4.4,10.1)$. Assuming that brightness and contrast are mutually independent, the probability that the image is natural is then expressed as:

$$
S N 1\left(\mathbf{I}_{L D R}\right)=\frac{1}{K\left(\mathbf{I}_{L D R}\right)} \times P d f_{\mathcal{N}}\left(\mathbf{I}_{L D R}\right) \times P d f_{\mathcal{B}}\left(\mathbf{I}_{L D R}\right)
$$

where $K$ is a factor used for the normalization, thus:

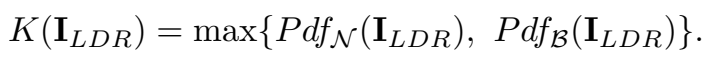

The TMQI1 expression is given by the combination of the two measures defined as:

$$
\begin{aligned}
T M Q I 1\left(\mathbf{I}_{H D R}, \mathbf{I}_{L D R}\right) & =a \times S F 1^{\alpha}\left(\mathbf{I}_{H D R}, \mathbf{I}_{L D R}\right) \\
& +(1-a) \times S N 1^{\beta}\left(\mathbf{I}_{L D R}\right),
\end{aligned}
$$

where the values of the parameters have been deduce as follows: $a=0.8012, \alpha=0.3046$ and $\beta=0.7088$ in an empirical way using 8 TMOs ("Reinhard" [8], "Drago" [7], "Durand" [10], "Mantiuk" [22], "Pattanaik" [23], and three remained TMOs in Adobe Photoshop namely "Exposure and Gamma", "Equalize Histogram" and "Local Adaptation" respectively) and a reduced database with 15 HDR test images whose dynamic range from $8 \mathrm{f}$-stops to $18 \mathrm{f}$-stops.

\section{Modified Tone Mapped image Quality Index}

Based on the TMQI1, Ma et al. [4] revised both of the terms and proposed a new version of the TMQI1 called TMQI2. Namely, the contrast visibility model for HDR images has been adapted to the local luminance. The estimate of the contrast in the HDR reference is therefore computed as the standard deviation in a patch divided by the local mean. However, the second term is modified much more severely.

\section{Feature Similarity Index for TM images}

The last full-reference metric is the Feature Similarity Index for TM images (called FSITM) [5]. It is based on the phase congruency features to calculate the difference between original and TM version of the the HDR image. More specifically, it 
computes the Locally Weighted Mean Phase Angle (LWMPA) to deduce the phase congruency. The main advantage of this feature is its robustness against noise.

\section{Performance AnAlysis of the REVISITED Visual QUALITY OBJECTIVE METRIC}

This section first compares the visual quality objective metric of the TM HDR images, described in the previous section, to the MOS. Discussions are provided in section III-B. Then, to improve the evaluation performance in accordance with the discussions provided in section III-B, section III-C revisits the selected objective metric so that to be consistent with the MOS.

\section{A. Evaluation context}

Note that during the visual evaluation the observers have no HDR display device (no-reference). The TM HDR images are displayed on the ColorEdge CG242W Color LCD monitor available in the lab with a good calibration in a random order during the testing period.

An observer assigns to each TM HDR image a mark ranging from 0 until 5: 5 for excellent, 4 very good, 3 good, 2 accepted, 1 unsatisfactory and 0 failed. The MOS table is obtained upon 26 observers. The chosen criteria is based on ability to recover details with natural ways.

Pearsons Linear Correlation Coefficient (PLCC) is selected to measure the relation between the objective visual quality metric of the TM image (i.e. Metric) and the MOS as follows:

$$
\begin{aligned}
& \text { PLCC = } \\
& \frac{\sum_{n=1}^{N} \sum_{m=1}^{M}(\operatorname{MOS}(n, m)-\overline{\mathbf{M O S}}) \cdot(\operatorname{Metric}(n, m)-\overline{\text { Metric }})}{\sqrt{\sum_{n=1}^{N} \sum_{m=1}^{M}(\operatorname{MOS}(n, m)-\overline{\mathbf{M O S}})^{2}}} \times \\
& \frac{1}{\sqrt{\sum_{n=1}^{N} \sum_{m=1}^{M}(\operatorname{Metric}(n, m)-\overline{\operatorname{Metric}})^{2}}}
\end{aligned}
$$

where $\overline{\mathrm{MOS}}$ and $\overline{\text { Metric }}$ are the mean values of two matrices MOS and Metric, respectively. $P L C C$ ranges from -1 and +1 .

Fifteen TMOs are used for the training such as "Drago" [7], "Reinhard" [8], "Ward" [9], "Durand" [10], "Tumblin" [11], "Schlick" [12], "Duan" [13], "Fattal" WRB [14], "Li" [15], "Husseis" [16], "NUHA" [19], "SEPENO" [17], "NONSEPENO" [18], "CEDP_Lin" [20] with $\beta=0.25$ and "CEDP_Opt" [20] with adaptive $\beta$. The training TM HDR image database is based on 24 HDR images with different dynamic range (or contrast ratio) from 7 f-stops to 29 f-stops, namely "Lausanne1", "CraterLake1", "Shasta2", "Synagogue", "Anturium", "BowRiver", "Bridges", "Stairway1", "ArchRock", "DollDoll", "ClockBuilding", "OxfordChurch", "BottlesSmall", "Montreal", "SmallOffice", "Light", "BridgeStudios2", "Memorial", "ClaridgeHotel", "Mistaya1", "BrookHouse", "PeaceRocks", "GGpark2" and "AtriumNight". Therefore the training database contains 360 TM HDR.

\section{B. Comparison of the Pearson Linear Correlation Coefficient using default parameters of the quality metrics}

Based on the evaluation context described above, one can notice that the PLCC associated with the TMQI1 [3] is highest (0.588) than those with TMQI2 [4] (0.512) and FSITM [5] (0.018). According to the provided results, the TMQI1 [3] (called TMQI_D) is retained but requires some changes. The parameters as tuned in the paper [3] are not well adapted since they have been trained only on a small set of TMOs (only 8) and a reduced test HDR images (only 15). Indeed this metric does not take into account the new distortions introduced by the new TMOs (e.g. "Duan" [13], "Fattal" WRB [14], "Li" [15], "Husseis" [16], "NUHA" [19], "SEPENO" [17], "NONSEPENO" [18], "CEDP" [20]). That is why this paper addresses, in section III-C, the question of re-evaluating the TMQI parameters to be in accordance with the MOS.

\section{Revised TMQI quality metric}

This section computes the best values of the parameters of the TMQI metric (i.e. $a, \alpha, \beta)$ in an iterative way as described in the Fig. 1. After the training process, the best values of the parameters are set to: $a=0.1, \alpha=0.1, \beta=0.2$ and $P L C C=0.7120$. One can notice that the Pearsons Linear Correlation Coefficient with the corresponding MOS increases from 0.588 (denoted TMQI_D for Default TMQI) to 0.7120 (denoted TMQI_P for revisited TMQI).
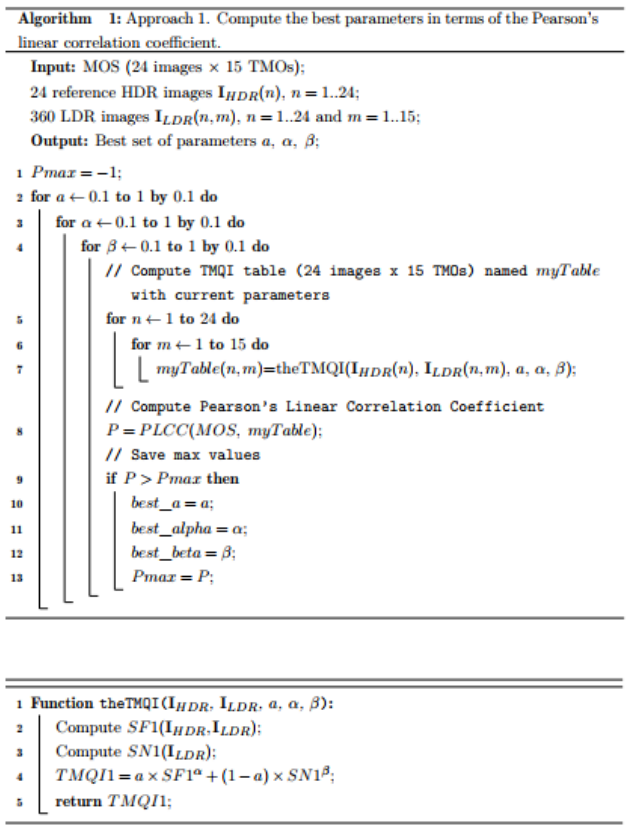

Fig. 1. Iterative algorithm to tune the TMQI parameters using PLCC criterion.

The Absolute Mean Error (AME) between the metric and normalized MOS (using the same training database) has been reduced from 0.3758 (TMQI_D) to 0.2626 (TMQI_P).

Fig. 2a and Fig. 2b show that the parameters are better designed on our training database ((a) "DollDoll" HDR test image (each group has 15 corresponding TMOs) as a column matrix, (b) "Ward" method (each group has 24 corresponding images) as a row matrix). The absolute mean errors are reduced for the image "DollDoll" and method "Ward".

Fig. 3 presents the TMQI metric versus the normalized MOS. One can observe that the values related to the points (TMQI_P, normalized MOS) are more gathered around the bisector line than the points (TMQI_D, normalized MOS). The more population of points near the bisector line the better the quality evaluation metric.

Fig. 4, Fig. 5, Fig. 6, Fig. 7 confirm the good choice of the tuned parameters when the visual evaluation is carried out on the TM HDR images taken from the training database.

\section{Simulation RESUltS}

This section evaluates the visual quality of the TM HDR images using the default TMQI and the revisited TMQI (i.e TMQI_P) 


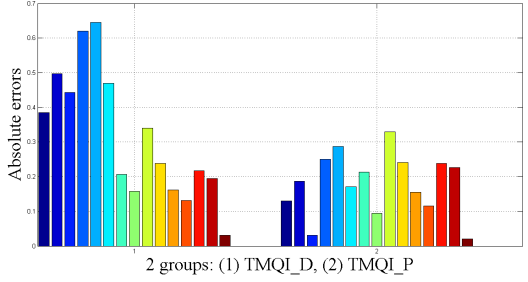

(a) "DollDoll" test HDR image (each group has 15 corresponding methods)

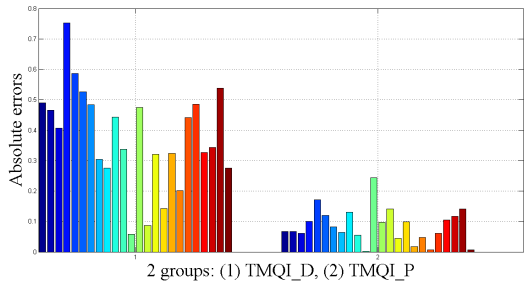

(b) "Ward" method (each group has 24 corresponding images)

Fig. 2. Absolute Mean Errors (AME) as one of our well-designed examples.
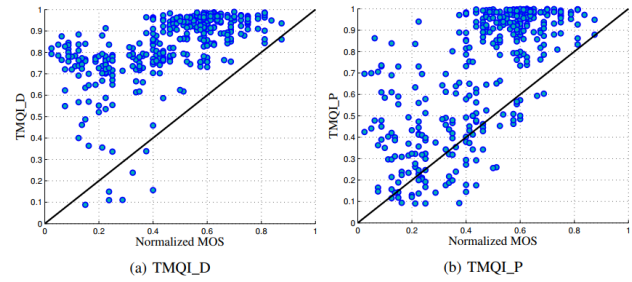

Fig. 3. TMQI_D and TMQI_P metrics versus the normalized MOS scores.

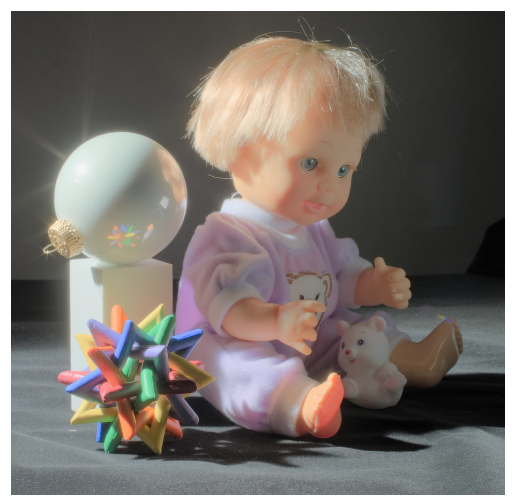

Fig. 4. "DollDoll" HDR test image (13.89 f-stops) - CEDP Opt with level 1 , Norm 1 with MOS=0.813, TMQI_D $=0.843$ and TMQI_P=0.808 (close to the MOS).

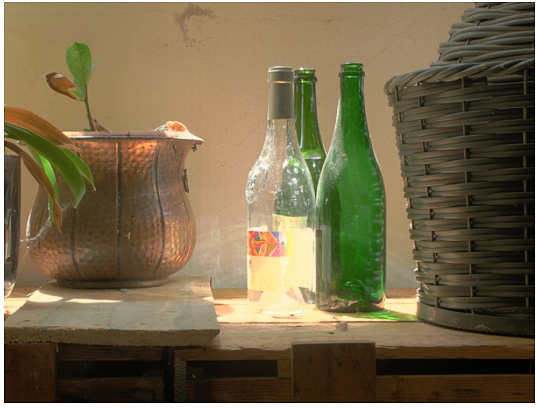

Fig. 5. "BottlesSmall" HDR test image (16.03 f-stops) - NONSEP with level 2 with MOS $=0.563$, TMQI_D $=0.903$ and TMQI $\mathrm{P}=0.889$ (close to the MOS).

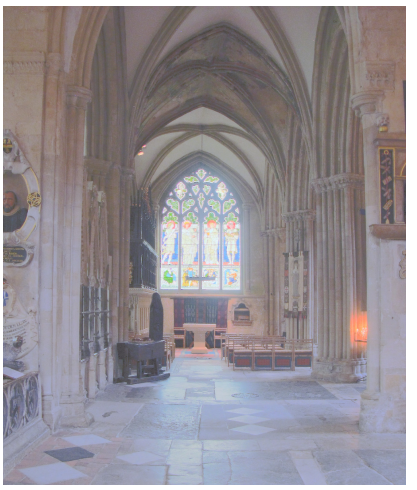

Fig. 6. "OxfordChurch" HDR test image (15.43 f-stops) - CEDP_Opt with level 1 , norm 1 with MOS $=0.738$, TMQI_D $=0.878$ and TMQI_P $=0.788$ (close to the MOS).
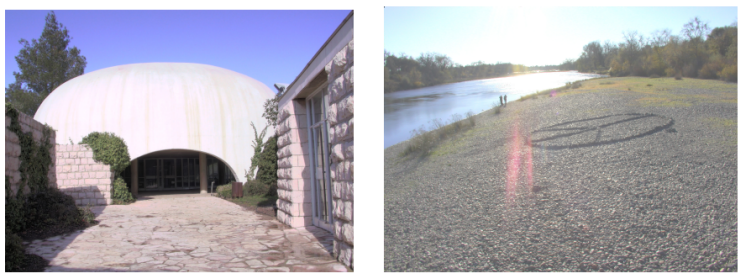

Fig. 7. Left: "Synagogue" HDR test image (8.57 f-stops) - CEDP_Lin with level 4 , norm 1 with MOS $=0.750$, TMQI_D $=0.908$ and TMQI_P $=0.869$ (close to the MOS), Right: "PeaceRocks" HDR test image (24.13 fstops) - SEPENOCA with level 1 with MOS $=0.613$, TMQI_D $=0.841$ and TMQI_P=0.788 (close to the MOS).

metrics. The resulting metrics are then compared to the MOS. Simulation results have been conducted on a large set of TM HDR images not belonging to the TM image training database to show the evaluation performance of our metric. TM "Lausanne", "WreathBuilding", "AtriumNight", "LondonChapel", "AdobeLobby" and "DomeBuilding" HDR images are selected to discuss the results. The respective TM images are provided by Fig. 8, Fig. 9, Fig. 10. One can observe that The visual quality evaluated by the revisited metric is closer to the MOS than the default TMQI metric.

\section{CONCLUSION}

This paper revised the TMQI objective metric to evaluate the visual quality of the tone mapped HDR images. The new values of the 

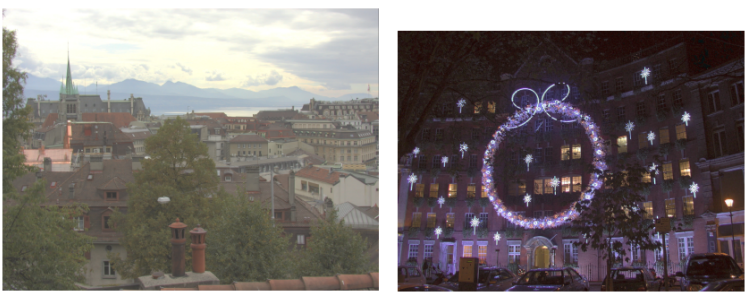

Fig. 8. Left: "Lausanne1" HDR test image (7.71 f-stops) - SEPENOCA with level 2 with MOS $=0.625$, TMQI $D=0.835$ and TMQI $\mathrm{P}=0.754$ (close to the MOS), Right: "WreathBuilding" HDR test image ( 23.88 f-stops) - Fattal RBW with MOS $=0.560$, TMQI_D $=0.908$ and TMQI_P $=0.589$ (close to the MOS).
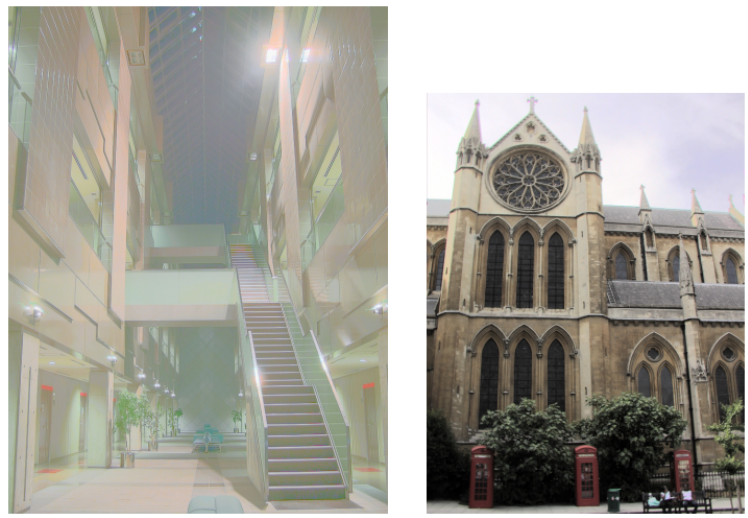

Fig. 9. Left: "AtriumNight" HDR test image (28.68 f-stops) - CEDP_Op with level 5 , norm 1 with MOS $=0.412$, TMQI_D $=0.768$ and TMQI_P $=0.562$ (close to the MOS); Right: "LondonChapel" HDR test image (14.19 f-stops) - Husseis with MOS=0.850, TMQI_D=0.722 and TMQI_P=0.853 (close to the MOS).

parameters have been deduced on a large TM training database taking into account many possible distorsions introduced on the TM HDR images. Simulation results show that this metric is strongly correlated with the MOS.

\section{REFERENCES}

[1] Aydin, Tunç Ozan and Mantiuk, Rafałand Myszkowski, Karol and Seidel, Hans-Peter, Dynamic range independent image quality assessment, ACM Trans. Graph, vol. 27, pp. 69:1-69:10, Aug. 2008.

[2] Mantiuk, R., and Myszkowski, K., and Seidel, H-P and Rogowitz, B., and Pappas, T., and Daly, S., Predicting Visible Differences in High Dynamic Range Images - Model and its Calibration, March 2005.

[3] Yeganeh, Hojatollah and Wang, Zhou, Objective Quality Assessment of Tone Mapped Images, Trans. Img. Proc., vol. 22, 2013, pp. 657-667.
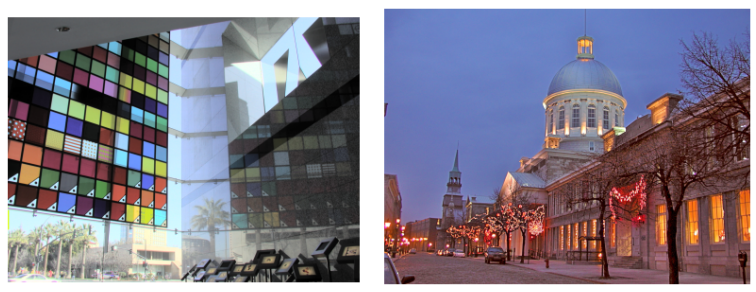

Fig. 10. Left: "AdobeLobby" HDR test image (16.95 f-stops) - Duan with MOS $=0.925$, TMQI_D $=0.738$ and TMQI_P $=0.947$ (close to the MOS), Right: "DomeBuilding" HDR test image (14.19 f-stops) - Li with MOS $=0.850$, TMQI_D $=0.922$ and TMQI_P=0.869 (close to the MOS).
[4] K. Ma and H. Yeganeh and K. Zeng and Z. Wang, High Dynamic Range Image Compression by Optimizing Tone Mapped Image Quality Index, IEEE Transactions on Image Processing, vol. 24, 2015, pp. 3086-3097.

[5] H. Ziaei Nafchi and A. Shahkolaei and R. Farrahi Moghaddam and M. Cheriet, FSITM: A Feature Similarity Index For Tone-Mapped Images, IEEE Signal Processing Letters, vol. 22, 2015, pp. 1026-1029.

[6] Banterle, F., Artusi, A., Debattista, K., and Chalmers, A., Advanced High Dynamic Range Imaging: Theory and Practice, AK Peters (now CRC Press), ISBN: 978-156881-719-4 (2011).

[7] Drago, F., Myszkowski, K., Annen, T., and Chiba, N., Adaptive Logarithmic Mapping for Displaying High Contrast Scenes, Computer Graphics Forum 22, pp. 419-426 (2003).

[8] Reinhard, E., and Devlin, K., Dynamic Range Reduction Inspired by Photoreceptor Physiology, IEEE Transactions on Visualization and Computer Graphics 11, pp. 13-24 (2005).

[9] Ward, G., Rushmeier, H., and Piatko, C., A Visibility Matching Tone Reproduction Operator for High Dynamic Range Scenes, IEEE Transactions on Visualization and Computer Graphics 3, pp. 291-306 (1997).

[10] Durand, F., and Dorsey, J., Fast Bilateral Filtering for The Display of High-dynamic-range Images, ACM Transactions on Graphics (TOG) Proceedings of ACM SIGGRAPH 21, pp. 257-266 (2002).

[11] Tumblin, J., and Rushmeier, H., Tone Reproduction for Realistic Images, IEEE Comput. Graph. Appl, pp. $42-48$ (1993).

[12] Schlick, C., Quantization Techniques for Visualization of High Dynamic Range Pictures, In Proceeding of the Fifth Eurographics Workshop on Rendering, pp. 7-18 (1994).

[13] Duan, J., Bressan, M., Dance, C., and Qiu, G., Tone-mapping High Dynamic Range Images by Novel Histogram Adjustment, Pattern Recognition, vol. 43, pp. 1847-1862 (2010).

[14] Fattal, R., Edge-Avoiding Wavelets and their Applications, ACM Trans. Graph (2009).

[15] Li, Y., Sharan, L., and Adelson, E.H., Compressing and Companding High Dynamic Range Images with Subband Architectures, ACM Trans. Graph. 24, pp. 836-844 (July 2005).

[16] Husseis, A., Mokraoui, A., and Matei, B., Revisited Histogram Equalization as HDR Images Tone Mapping Operators, 17th IEEE International Symposium on Signal Processing and Information Technology, 2017.

[17] Thai, B.C., Mokraoui, A., and Matei, B., Performance Evaluation of High Dynamic Range Image Tone Mapping Operators Based on Separable Non-linear Multiresolution Families, 24th European Signal Processing Conference, pp. 1891-1895 (August 2016).

[18] Thai, B.C., Mokraoui, A., and Matei, B., Image Tone Mapping Approach Using Essentially Non-Oscillatory Bi-quadratic Interpolations Combined with a Weighting Coefficients Strategy, 17th IEEE International Symposium on Signal Processing and Information Technology, 2017.

[19] Thai, B.C., Mokraoui, A., and Matei, B., Piecewise linear perceptual quantizer as a non-uniform histogram equalization adjustment for contrast enhancement of tone mapped HDR images, 9th International Symposium on Signal, Image, Video and Communications, 2018.

[20] Thai, B.C., Mokraoui, A., and Matei, B., Contrast Enhancement and Details Preservation of Tone Mapped High Dynamic Range Images, Journal of Visual Communication and Image Representation, (2018).

[21] Z. Wang, E. P. Simoncelli, and A. C. Bovik., Multiscale structural similarity for image quality assessment, Journal of Visual Communication and Image Representation, 37th Asilomar Conference on Signals, Systems Computers, (2003).

[22] Rafal Mantiuk, Karol Myszkowski, and Hans-Peter Seidel. A Perceptual Framework for Contrast Processing of High Dynamic Range Images. In: ACM Trans. Appl. Percept. (June 2006), pp. 286308

[23] Sumanta N. Pattanaik et al. Time-dependent Visual Adaptation for Fast Realistic Image Display. In: Pro. 27th Annual Con. on Computer Graphics and Interactive Techniques. SIGGRAPH 00, 2000, pp. 4754

[24] Andrew B. Watson. Visual Detection of Spatial Contrast Patterns: Evaluation of Five Simple Models In: Opt. Express 6.1 (Jan. 2000), pp. 1233

[25] W. J. Crozier, ed. On the Variability of Critical Illumination for Flicker Fusion and Intensity Discrimination, 1935

[26] J. Mannos and D. Sakrison. The Effects of a Visual Fidelity Criterion of the Encoding of Images, In: IEEE Trans. Inf. Theor. 20.4 (Sept. 2006), pp. 525536

[27] D. H. Kelly. Effects of Sharp Edges on the Visibility of Sinusoidal Gratings. In: Journal of the Optical Society of America (1917-1983) 60 (Jan. 1970), p. 98 